\author{
Professor Zongrun WANG, PhD \\ E-mail: zrwang0209@sina.com \\ School of Business \\ Central South University, China \\ Tangtang HE \\ E-mail: 1294134835@qq.com \\ Xuekai NI \\ E-mail: 13111866959@163.com \\ School of Business \\ Central South University, China
}

\title{
A PORTFOLIO OPTIMIZATION PERSPECTIVE BASED ON TRI- REFERENCE POINT THEORY FOR EXPLAINING THE ATTRACTIVENESS OF STRUCTURED PRODUCTS
}

\begin{abstract}
In tri-reference point theory, four regions-failure, loss, gain and success - are formed by three reference points-minimum requirement (MR), status quo $(S Q)$, and goal $(G)$-which play an important role in decision-making. Considering investors' three reference points with respect to investment returns, optimization models of portfolios including structured products are constructed by employing investors' maximum perceived value in tri-reference point theory as the objective under the premise of satisfying the safety-first principle. Then, a hybrid particle swarm optimization algorithm that is suitable for solving these models is designed. In this setting, the superiority of structured products relative to riskless assets and underlying assets are studied by changing the parameters in trireference point theory. The results show that structured products are the most favored by investors when both $M R$ and $G$ are relatively high. This is because under these circumstances, structured products are capable of satisfying investors' demand for relatively high returns under the premise of ensuring safety and in turn attract the most investors in the market.
\end{abstract}

Keywords: Portfolio selection, Structured product, Tri-reference point theory, Particle swarm optimization.

\section{JEL Classification: G11}

\section{Introduction}

Structured products represent one of the most important financial innovations for retail investors developed during the last decade (Entrop et al., 2016). They are securities derived from or based on a single security, a basket of

DOI: 10.24818/18423264/54.1.20.12 
securities, an index, a commodity, a debt issuance and/or a foreign currency and offer investors an alternative choice of a diversified portfolio. They are a mixture of two asset classes and typically issued in the form of a corporate bond or a certificate of deposit, but instead of having a predetermined rate of interest, their payoff depends on the performance of an underlying asset class.

Given the craze for structured products, many scholars have investigated the reasons for their sudden rise. Kiriakopoulos and Mavralexakis (2011) propose that a low interest rate environment stimulates investors' desire for high returns. A survey by Rieger and Hens (2012) suggests that the demand for capital-protected products can be explained by loss aversion and saving motives. Abreu and Mendes (2018) show that gambling appears to be an important motivation for trading and that overconfidence drives more trading in structured products with the help of information from one of the top five Portuguese banks. Moreover, they confirm that financial knowledge and bank advice are positively associated with investment in more complex products. Rieger (2012) stresses the influence of systematic probability misestimation on complex financial investment decisions in the context of structured products. Other research focuses on the application of behavioral utility functions. Hens et al. explain purchasing behavior with respect to structured products via prospect theory, and they emphasize that in the face of certain loss, investors' risk-seeking psychology is a key factor (Hens \& Rieger, 2008; Hens \& Rieger, 2014). The empirical results in Döbeli and Vanini (2010) show that participants' behaviors do not conform to expected utility theory but can be described by behavioral finance models. Vandenbroucke (2015) analyzes the conditions that lead to the emergence of structured products by constructing a portfolio and finds that the probability distortion feature in cumulative prospect theory offers a rational explanation for their existence.

The abovementioned literature is all based on the psychological perceptions of investors, describing the behavioral biases affecting their decisionmaking process, and the quantitative studies are mostly limited to interpreting the allure of structured products from the perspective of prospect theory. In general, their investigations of investors' purchasing behavior for structured products are far from sufficient.

Investors determine the optimal portfolio for increasing investment returns and reducing investment risk. Markowitz (1952) inaugurated a stream of research on portfolio theory, using expected utility theory as the cornerstone and variance as a risk measure. However, over time, scholars have gradually discovered the limitations of the mean-variance model.

First, it is difficult for expected utility theory to explain the Allais paradox (Allais, 1953) and framing and reversal effects (Kahneman \& Tversky, 1979). Second, the mean-variance model assumes that the goal of decision-making is to maximize total wealth. In contrast, investment behavior often manifests as bounded rationality. The goal of investors is not to maximize total wealth but to maximize perceived return. Based on this understanding, a reference point becomes the 
A Portfolio Optimization Perspective Based on Tri-Reference Point Theory for Explaining the Attractiveness of Structured Products

decision benchmark in prospect theory proposed by Tversky and Kahneman (1992). In subsequent work, Lopes (1987) develops SP/A theory, in which decisions depend on the agent's "security" and "potential". Benefiting from the foundational theories of their predecessors, Wang and Johnson (2012) propose trireference point (TRP) theory with minimum requirement (MR), status quo (SQ), and goal $(\mathrm{G})$ as the reference points for decision makers, which is the theoretical tool that this paper emphasizes. Although TRP theory is a recent innovation, it has been widely used in various areas related to decision-making management, such as gamified crowdsourcing tasks (Harris \& Wu, 2014), salary perception (Zhao et al., 208), HR management (Hu \& Wang, 2014), food choice (Lagerkvist et al., 2015), and even international second home retirement motives in Malaysia (Keemun \& Musa, 2015).

In the risk measurement field, the mean-variance model has been called into question because it treats upside and downside differences as equivalent. However, in fact, investors usually pay attention only to downside risk. Value at risk ( $\mathrm{VaR})$ is a commonly employed downside risk measure. Note that with respect to investment behavior, investors generally have their own risk tolerance and seek to maximize their utility above the MR. The safety-first principle proposed by Roy argues that investors will attempt to reduce the probability of being below a "disaster" level (Roy, 1952). Pyle and Turnovsky (1970) summarize three forms of the safety-first principle. Based on the third form, we optimize portfolios that include structured products. In substance, this also exploits the idea of downside risk and considers more investor psychology factors. We define the MR in TRP theory as the "disaster" level, take the probability of being below it as an investor's risk tolerance, and set the maximization of the TRP value as the optimization goal. Levy and Levy (2009) both theoretically and experimentally demonstrate that the safety-first principle plays an important role in decision-making. Safety first has been widely used in investment decision-making (Yao et al., 2015; Gao et al., 2016; Chiu et al., 2018).

The algorithms required for various practical portfolio optimization problems have been studied extensively. Nalpas et al. (2017) develop a MonteCarlo-FDH algorithm in a multi-moment setting. Macedo et al. (2017) study the use of multi-objective evolutionary algorithms in portfolio optimization within a mean-semivariance framework. Liagkouras (2019) introduce a new threedimensional encoding multi-objective evolutionary algorithm for the cardinality constrained portfolio optimization problem. However, efficient (not heuristic) algorithms to solve the portfolio selection model with an objective function that includes limits of integrals with unknown decision variables have rarely been developed. This is the most prominent feature of our proposed models. To solve this problem efficiently, we design a hybrid particle swarm optimization algorithm inspired by Zhang et al. (2016).

In summary, the main contributions of this paper are the following: (1) the integration of TRP theory with the safety-first principle and the development of

DOI: 10.24818/18423264/54.1.20.12 
novel models for portfolio selection that can be used to analyze structured products' role in investment portfolios and explain why they are favored by investors; (2) the development of a hybrid particle swarm optimization algorithm that takes into account both the speed and accuracy of the solving process.

This paper is organized as follows. In the next section, we introduce TRP theory and the safety-first principle. We then place structured products in the portfolio and build portfolio selection models in Section 3. Subsequently, we design a new algorithm to solve the problem in Section 4. In Section 5, we analyze the reasons that and conditions under which investors prefer structured products. A summary and an extended discussion are provided in Section 6.

\section{Basis of theories}

\subsection{Tri-reference point theory}

TRP theory is a novel method used to explain a series of risky choice behaviors. It divides the perceptual basis employed by decision makers into three criteria-MR, $\mathrm{SQ}$, and $\mathrm{G}$ - and divides risky outcomes into four functional regions: failure (below MR), loss (between MR and SQ), gain (between SQ and G), and success (above G). The core of TRP theory is that when the outcome is located in different regions, the perception intensity of decision makers is different. Specifically, the disutility of a failure is greater than the utility of a success, the utility of a success is greater than the disutility of a loss, and the disutility of a loss is greater than the utility of a gain. Compared with expected utility theory, TRP theory can explain the Allais paradox and framing and reversal effects. Compared with prospect theory, TRP theory enriches the risky perception criteria of decision makers and restores the authenticity of probability.

Use the notation $\left(x_{1}, p_{1} ; \cdots ; x_{n}, p_{n}\right)$ to signify an n-outcome gamble offering an outcome with probability $p_{i}$. Let $x_{1}<\cdots<x_{n}$ and $x_{i}<M R<x_{i+1}<\cdots<x_{j}<S Q<x_{j+1}<\cdots<x_{k}<G<x_{k+1}$. When SQ=0, the TRP value is expressed as follows (Wang \& Johnson, 2012):

$$
T R P=\beta_{F} \cdot M R \sum_{m=1}^{i} p_{m}+\sum_{m=i+1}^{j} p_{m} \cdot \beta_{-} \cdot x_{m}+\sum_{m=j+1}^{k} p_{m} \cdot \beta_{+} \cdot x_{m}+\beta_{S} \cdot G \sum_{m=k+1}^{n} p_{m}
$$

where $\beta_{F}, \beta_{-}, \beta_{+}$, and $\beta_{S}$ represent the failure weight, loss weight, gain weight and success weight, respectively, satisfying $0<\beta_{+}<\beta_{-}<\beta_{S}<\beta_{F}$. Note that in the model, when MR and $\mathrm{G}$ are fixed, the perception values of all points below MR or above $\mathrm{G}$ are the same.

Assuming that the MR, SQ and G of an investor in an investment activity are fixed, the TRP value function is obtained, as shown in Figure 1. 
A Portfolio Optimization Perspective Based on Tri-Reference Point Theory for Explaining the Attractiveness of Structured Products

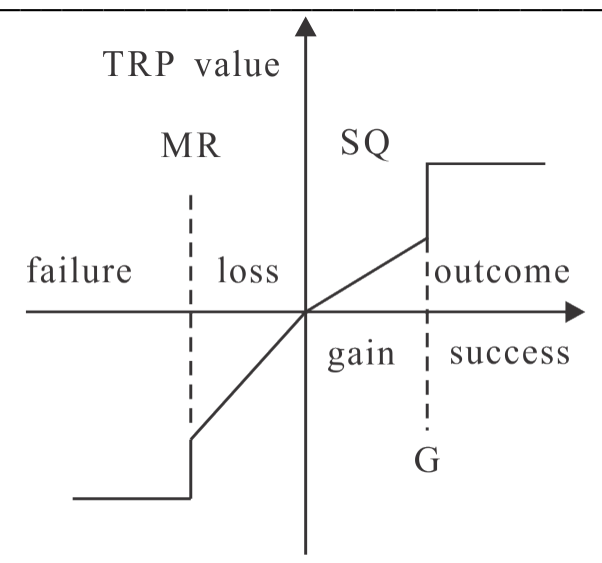

Figure 1.TRP value function

The discrete model expressed in equation (1) is extended to a continuous function model as follows:

$T R P=\beta_{F} \cdot M R \int_{-\infty}^{M R} f(z) d z+\beta_{-} \int_{M R}^{S Q} z \cdot f(z) d z+\beta_{+} \int_{S Q}^{G} z \cdot f(z) d z+\beta_{S} \cdot G \int_{G}^{+\infty} f(z) d z$

where $z$ represents the outcome, and $f(z)$ is the probability density function of $z$. This model concisely reflects the different impacts on decision makers when the outcome, also called the objective value, is located in different regions.

\subsection{Safety-first principle}

The so-called safety-first principle, as the name suggests, places the MR in the most important position. Pyle \& Turnovsky (1970) summarize the following three forms of safety-first principle, where $z$ is the return, and $\bar{z}$ is the disaster level: (1) Minimize the probability that the return is below the disaster level. That is, min $P_{r}(z \leq \bar{z})$. (2) Maximize the disaster level on the condition that the probability of a return below the disaster level is less than a certain probability $\sigma$. That is, max $\bar{z}$ subject to $P_{r}(z \leq \bar{z}) \leq \sigma$. (3) Maximize the expected return on the condition that the probability of a return below the disaster level is less than a certain probability $\sigma$. That is, $\max \mu$ subject to $P_{r}(z \leq \bar{z}) \leq \sigma$.

We apply the third form of the safety-first principle, replacing the expected value with the TRP value to describe investors' psychological value. Because the MR of TRP theory has the same meaning as the "disaster level" of the safety-first principle, that is, the maximum loss an individual can sustain, the two are equated. $z$ represents the objective value, and the constraint is rewritten as follows:

$$
\int_{-\infty}^{M R} f(z) d z \leq \sigma
$$

\section{DOI: $10.24818 / 18423264 / 54.1 .20 .12$}




\section{Model building}

\subsection{Problem description}

To investigate the advantages of structured products over riskless assets and underlying assets, that is, the reasons that and conditions under which structured products attract investors more than riskless assets and underlying assets, we construct a portfolio containing these three products: asset 1, a riskless asset with a fixed annual return $\mathrm{R}_{1} \mathrm{of} 5 \%$; asset 2 , a risky asset with expected return and volatility of $10 \%$ and $20 \%$, respectively; and asset 3, a structured product whose payoff depends on the performance of asset 2, which 2 is also called the underlying asset of asset 3. Suppose that all assets are held for one year.

Regarding asset 2, some empirical studies show that the return on financial assets can be described by a t-distribution, with a better fit when there are 5 or 6 degrees of freedom. Therefore, let the return follow a transformed t distribution $($ Cao, 2017) with 6 degrees of freedom, a location parameter of $10 \%$, and a scale parameter of $20 \%$. For asset 3, we analyze two types of interval structured products commonly seen in China's financial market. The description of the first product's (3A's) payoff is divided into two parts: (1) When the underlying return $R_{2}$ is greater than or equal to $a$, the payoff is $b$. (2) When the underlying return $\mathrm{R}_{2}$ is less than $a$, the payoff is $c$. The description of the second product payoff is divided into three parts: (1) When the underlying return $\mathrm{R}_{2}$ is greater than $d$, the payoff is $d$. (2) When the underlying return $\mathrm{R}_{2}$ is less than $e$, the payoff is $e$. (3) When the underlying return $\mathrm{R}_{2}$ is located between $e$ and $d$, the payoff is $\mathrm{R}_{2}$. The payoff functions of products $3 \mathrm{~A}$ and $\mathrm{BB}$ are as follows:

$$
R(3 A)=\left\{\begin{array}{ll}
b & R_{2} \geq a \\
c & R_{2}<a
\end{array} R(3 B)= \begin{cases}d & R_{2}>d \\
R_{2} & e \leq R_{2} \leq d \\
e & R_{2}<e\end{cases}\right.
$$

Because the expected annual return of asset 2 is $10 \%$, when the threshold value $a$ of the underlying return of product $3 \mathrm{~A}$ is set at $10 \%$, the probabilities of the two possible payoffs of product $3 \mathrm{~A}$ are $50 \%$. To make it comparable to asset 1 , the expected annual return of product $3 \mathrm{~A}$ should be set at $5 \%$, which is the same as that of asset 1. For actual structured product payoff boundaries in the financial market, $1 \%-9 \%$ is common. Therefore, the upper limit payoff $b$ and the lower limit $c$ of product $3 \mathrm{~A}$ are set at $7 \%$ and $3 \%$, respectively. Using settings of $8 \%$ and $2 \%$ or $9 \%$ and $1 \%$ will only change the specific values of the results slightly and will not affect the conclusions of this paper. Similarly, for product 3B, the lower limit $e$ of the payoff is set at $3 \%$, which is the same as that of product $3 \mathrm{~A}$. To make it have the same expected return as asset 1, the upper limit $d$ is set at $6.21 \%$.

\subsection{Portfolio selection model}

In investment activities, the MR in TRP theory can be regarded as the tolerance limits set by investors according to their own wealth level or 
A Portfolio Optimization Perspective Based on Tri-Reference Point Theory for Explaining the Attractiveness of Structured Products

psychological characteristics. In addition, the MR can also be interpreted as different maximum loss lines for different fields in which investors invest for different purposes. The SQ reflects the current state of investors, set at 0 , indicating that the basic state of investors is the "inaction" state, and the safest investment is "no investment". G represents the highest return that investors believe can be achieved. Moreover, the premise of maximizing psychological value is that the investment risk satisfies the individual's tolerance, that is, the probability of obtaining a return below the tolerance must be less than the investor's preset probability. After ensuring security, the optimization of psychological value can be discussed. Based on this, a portfolio selection model that is in line with an investor's psychological perception is established by combining TRP theory and the safety-first principle.

\subsubsection{The model of portfolio $P 1$ including structured product $3 \mathrm{~A}$}

Set $\theta=\left(\theta_{1}, \theta_{2}, \theta_{3}\right)$ as the vector of the investment proportions of asset 1 , asset 2 and asset 3 ; the payoff of $\mathrm{P} 1$ is the following:

$$
Z= \begin{cases}R_{1} \cdot \theta_{1}+R_{2} \cdot \theta_{2}+b \cdot \theta_{3} & R_{2} \geq a \\ R_{1} \cdot \theta_{1}+R_{2} \cdot \theta_{2}+c \cdot \theta_{3} & R_{2}<a\end{cases}
$$

Let $p(\cdot)$ be the density function of the transformed $\mathrm{t}$ distribution mentioned above; then the density function of $\mathrm{Z}$ is as follows:

$$
f(z)= \begin{cases}\frac{1}{\theta_{2}} \cdot p\left(\frac{z-R_{1} \cdot \theta_{1}-b \cdot \theta_{3}}{\theta_{2}}\right) & z \geq N_{2} \\ \frac{1}{\theta_{2}} \cdot p\left(\frac{z-R_{1} \cdot \theta_{1}-c \cdot \theta_{3}}{\theta_{2}}\right) & z<N_{1}\end{cases}
$$

where $N_{1}=\mathrm{R}_{1} \cdot \theta_{1}+a \cdot \theta_{2}+c \cdot \theta_{3}, N_{2}=\mathrm{R}_{1} \cdot \theta_{1}+a \cdot \theta_{2}+b \cdot \theta_{3}$.

Since the density function $f(z)$ is a piecewise function, we construct the portfolio selection models in different cases.

Case 1

$$
\begin{aligned}
\operatorname{Min}-F_{1}(\boldsymbol{\theta}) & \\
=- & \beta_{F} \cdot M R \int_{-\infty}^{M R} \frac{1}{\theta_{2}} \cdot p\left(\frac{z-R_{1} \cdot \theta_{1}-c \cdot \theta_{3}}{\theta_{2}}\right) d z-\beta_{-} \int_{M R}^{S Q} z \cdot \frac{1}{\theta_{2}} \cdot p\left(\frac{z-R_{1} \cdot \theta_{1}-c \cdot \theta_{3}}{\theta_{2}}\right) d z \\
& -\beta_{+} \int_{S Q}^{N_{1}} z \cdot \frac{1}{\theta_{2}} \cdot p\left(\frac{z-R_{1} \cdot \theta_{1}-c \cdot \theta_{3}}{\theta_{2}}\right) d z-\beta_{+} \int_{N_{2}}^{G} z \cdot \frac{1}{\theta_{2}} \cdot p\left(\frac{z-R_{1} \cdot \theta_{1}-b \cdot \theta_{3}}{\theta_{2}}\right) d z \\
& -\beta_{s} \cdot G \cdot \int_{G}^{+\infty} \frac{1}{\theta_{2}} \cdot p\left(\frac{z-R_{1} \cdot \theta_{1}-b \cdot \theta_{3}}{\theta_{2}}\right) d z
\end{aligned}
$$

S.t. $N_{2} \leq G$

DOI: 10.24818/18423264/54.1.20.12 
Zongrun Wang, Tangtang He, Xuekai Ni

$$
\begin{aligned}
& \int_{-\infty}^{M R} \frac{1}{\theta_{2}} \cdot p\left(\frac{z-R_{1} \cdot \theta_{1}-c \cdot \theta_{3}}{\theta_{2}}\right) d z \leq \sigma \\
& \theta_{1}+\theta_{2}+\theta_{3}=1 \\
& 0 \leq \theta_{i} \leq 1, \quad i \in\{1,2,3\}
\end{aligned}
$$

Case 2

$\operatorname{Min}-F_{2}(\boldsymbol{\theta})$

S.t. $N_{1} \leq G$

$$
\begin{aligned}
= & -\beta_{F} \cdot M R \int_{-\infty}^{M R} \frac{1}{\theta_{2}} \cdot p\left(\frac{z-R_{1} \cdot \theta_{1}-c \cdot \theta_{3}}{\theta_{2}}\right) d z-\beta_{-} \int_{M R}^{S Q} z \cdot \frac{1}{\theta_{2}} \cdot p\left(\frac{z-R_{1} \cdot \theta_{1}-c \cdot \theta_{3}}{\theta_{2}}\right) d z \\
& -\beta_{+} \int_{S Q}^{N_{1}} z \cdot \frac{1}{\theta_{2}} \cdot p\left(\frac{z-R_{1} \cdot \theta_{1}-c \cdot \theta_{3}}{\theta_{2}}\right) d z-\beta_{s} \cdot G \int_{N_{2}}^{-\infty} \frac{1}{\theta_{2}} \cdot p\left(\frac{z-R_{1} \cdot \theta_{1}-b \cdot \theta_{3}}{\theta_{2}}\right) d z
\end{aligned}
$$

$$
\begin{aligned}
& N_{2}>G \\
& \int_{-\infty}^{M R} \frac{1}{\theta_{2}} \cdot p\left(\frac{z-R_{1} \cdot \theta_{1}-c \cdot \theta_{3}}{\theta_{2}}\right) d z \leq \sigma \\
\theta_{1}+\theta_{2}+\theta_{3}=1 & \\
0 \leq \theta_{i} \leq 1, \quad i \in\{1,2,3\} &
\end{aligned}
$$

Case 3

$$
\begin{aligned}
& \text { Min }- F_{3}(\boldsymbol{\theta}) \\
&=-\beta_{F} \cdot M R \int_{-\infty}^{M R} \frac{1}{\theta_{2}} \cdot p\left(\frac{z-R_{1} \cdot \theta_{1}-c \cdot \theta_{3}}{\theta_{2}}\right) d z-\beta_{-} \int_{M R}^{S Q} z \cdot \frac{1}{\theta_{2}} \cdot p\left(\frac{z-R_{1} \cdot \theta_{1}-c \cdot \theta_{3}}{\theta_{2}}\right) d z \\
&-\beta_{+} \int_{S Q}^{G} z \cdot \frac{1}{\theta_{2}} \cdot p\left(\frac{z-R_{1} \cdot \theta_{1}-c \cdot \theta_{3}}{\theta_{2}}\right) d z-\beta_{\mathrm{s}} \cdot G \int_{G}^{N_{1}} \frac{1}{\theta_{2}} \cdot p\left(\frac{z-R_{1} \cdot \theta_{1}-c \cdot \theta_{3}}{\theta_{2}}\right) d z \\
&-\beta_{\mathrm{s}} \cdot G \int_{N_{2}}^{+\infty} \frac{1}{\theta_{2}} \cdot p\left(\frac{z-R_{1} \cdot \theta_{1}-b \cdot \theta_{3}}{\theta_{2}}\right) d z
\end{aligned}
$$

S.t. $N_{1}>G$

$$
\begin{gathered}
\int_{-\infty}^{M R} \frac{1}{\theta_{2}} \cdot p\left(\frac{z-R_{1} \cdot \theta_{1}-c \cdot \theta_{3}}{\theta_{2}}\right) d z \leq \sigma \\
\theta_{1}+\theta_{2}+\theta_{3}=1 \\
0 \leq \theta_{i} \leq 1, \quad i \in\{1,2,3\}
\end{gathered}
$$


A Portfolio Optimization Perspective Based on Tri-Reference Point Theory for Explaining the Attractiveness of Structured Products

\subsubsection{The model of portfolio $\mathbf{P} 2$ including structured product $3 \mathrm{~B}$}

$$
\text { The payoff of } \mathrm{P} 2 \text { is } Z= \begin{cases}R_{1} \cdot \theta_{1}+R_{2} \cdot \theta_{2}+d \cdot \theta_{3} & R_{2}>d \\ R_{1} \cdot \theta_{1}+\left(\theta_{2}+\theta_{3}\right) \cdot R_{2} & e \leq R_{2} \leq d \text { and the } \\ R_{1} \cdot \theta_{1}+R_{2} \cdot \theta_{2}+e \cdot \theta_{3} & R_{2}<e,\end{cases}
$$

density function of $\mathrm{Z}$ is:

$$
f(z)= \begin{cases}\frac{1}{\theta_{2}} \cdot p\left(\frac{z-R_{1} \cdot \theta_{1}-d \cdot \theta_{3}}{\theta_{2}}\right) & z>N_{4} \\ \frac{1}{\theta_{2}+\theta_{3}} \cdot p\left(\frac{z-R_{1} \cdot \theta_{1}}{\theta_{2}+\theta_{3}}\right) & N_{3} \leq z \leq N_{4} \\ \frac{1}{\theta_{2}} \cdot p\left(\frac{z-R_{1} \cdot \theta_{1}-e \cdot \theta_{3}}{\theta_{2}}\right) & z<N_{3}\end{cases}
$$

where $N_{3}=\mathrm{R}_{1} \cdot \theta_{1}+e \cdot\left(\theta_{2}+\theta_{3}\right), N_{4}=\mathrm{R}_{1} \cdot \theta_{1}+d \cdot\left(\theta_{2}+\theta_{3}\right)$. The portfolio selection models in the different cases are constructed as follows.

Case 4

$$
\begin{aligned}
& \text { Min }- F_{4}(\boldsymbol{\theta}) \\
&=-\beta_{F} \cdot M R \int_{-\infty}^{M R} \frac{1}{\theta_{2}} \cdot p\left(\frac{z-R_{1} \cdot \theta_{1}-e \cdot \theta_{3}}{\theta_{2}}\right) d z-\beta_{-} \int_{M R}^{S Q} z \cdot \frac{1}{\theta_{2}} \cdot p\left(\frac{z-R_{1} \cdot \theta_{1}-e \cdot \theta_{3}}{\theta_{2}}\right) d z \\
& \quad-\beta_{+} \int_{S Q}^{N_{3}} z \cdot \frac{1}{\theta_{2}} \cdot p\left(\frac{z-R_{1} \cdot \theta_{1}-e \cdot \theta_{3}}{\theta_{2}}\right) d z-\beta_{+} \int_{N_{3}}^{N_{4}} z \cdot \frac{1}{\theta_{2}+\theta_{3}} \cdot p\left(\frac{z-R_{1} \cdot \theta_{1}}{\theta_{2}+\theta_{3}}\right) d z \\
&-\beta_{+} \int_{N_{4}}^{G} z \cdot \frac{1}{\theta_{2}} \cdot p\left(\frac{z-R_{1} \cdot \theta_{1}-d \cdot \theta_{3}}{\theta_{2}}\right) d z-\beta_{\mathrm{s}} \cdot G \int_{G}^{+\infty} \frac{1}{\theta_{2}} \cdot p\left(\frac{z-R_{1} \cdot \theta_{1}-d \cdot \theta_{3}}{\theta_{2}}\right) d z
\end{aligned}
$$

S.t. $N_{4} \leq G$

$$
\begin{gathered}
\int_{-\infty}^{M R} \frac{1}{\theta_{2}} \cdot p\left(\frac{z-R_{1} \cdot \theta_{1}-e \cdot \theta_{3}}{\theta_{2}}\right) d z \leq \sigma \\
\theta_{1}+\theta_{2}+\theta_{3}=1 \\
0 \leq \theta_{i} \leq 1, \quad i \in\{1,2,3\}
\end{gathered}
$$




\section{Case 5}

$$
\begin{aligned}
& \operatorname{Min}-F_{5}(\boldsymbol{\theta}) \\
& =-\beta_{F} \cdot M R \int_{-\infty}^{M R} \frac{1}{\theta_{2}} \cdot p\left(\frac{z-R_{1} \cdot \theta_{1}-e \cdot \theta_{3}}{\theta_{2}}\right) d z-\beta_{-} \int_{M R}^{S Q} z \cdot \frac{1}{\theta_{2}} \cdot p\left(\frac{z-R_{1} \cdot \theta_{1}-e \cdot \theta_{3}}{\theta_{2}}\right) d z \\
& -\beta_{+} \int_{S Q}^{N_{3}} z \cdot \frac{1}{\theta_{2}} \cdot p\left(\frac{z-R_{1} \cdot \theta_{1}-e \cdot \theta_{3}}{\theta_{2}}\right) d z-\beta_{+} \int_{N_{3}}^{G} z \cdot \frac{1}{\theta_{2}+\theta_{3}} \cdot p\left(\frac{z-R_{1} \cdot \theta_{1}}{\theta_{2}+\theta_{3}}\right) d z \\
& -\beta_{\mathrm{s}} \cdot G \int_{G}^{N_{4}} \frac{1}{\theta_{2}+\theta_{3}} \cdot p\left(\frac{z-R_{1} \cdot \theta_{1}}{\theta_{2}+\theta_{3}}\right) d z-\beta_{\mathrm{s}} \cdot G \int_{N_{4}}^{+\infty} \frac{1}{\theta_{2}} \cdot p\left(\frac{z-R_{1} \cdot \theta_{1}-d \cdot \theta_{3}}{\theta_{2}}\right) d z \\
& N_{4} \geq G \\
& I(\boldsymbol{\theta})=\int_{-\infty}^{M R} \frac{1}{\theta_{2}} \cdot p\left(\frac{z-R_{1} \cdot \theta_{1}-e \cdot \theta_{3}}{\theta_{2}}\right) d z \leq \sigma \\
& \theta_{1}+\theta_{2}+\theta_{3}=1 \\
& 0 \leq \theta_{i} \leq 1, \quad i \in\{1,2,3\}
\end{aligned}
$$

Case 6

$$
\begin{aligned}
& \operatorname{Min}-F_{6}(\boldsymbol{\theta}) \\
& =-\beta_{F} \cdot M R \int_{-\infty}^{M R} \frac{1}{\theta_{2}} \cdot p\left(\frac{z-R_{1} \cdot \theta_{1}-e \cdot \theta_{3}}{\theta_{2}}\right) d z-\beta_{-} \int_{M R}^{S Q} z \cdot \frac{1}{\theta_{2}} \cdot p\left(\frac{z-R_{1} \cdot \theta_{1}-e \cdot \theta_{3}}{\theta_{2}}\right) d z \\
& -\beta_{+} \int_{S Q}^{G} z \cdot \frac{1}{\theta_{2}} \cdot p\left(\frac{z-R_{1} \cdot \theta_{1}-e \cdot \theta_{3}}{\theta_{2}}\right) d z-\beta_{\mathrm{s}} \cdot G \int_{G}^{N_{3}} \frac{1}{\theta_{2}} \cdot p\left(\frac{z-R_{1} \cdot \theta_{1}-e \cdot \theta_{3}}{\theta_{2}}\right) d z \\
& -\beta_{s} \cdot G \int_{N_{3}}^{N_{4}} \frac{1}{\theta_{2}+\theta_{3}} \cdot p\left(\frac{z-R_{1} \cdot \theta_{1}}{\theta_{2}+\theta_{3}}\right) d z-\beta_{\mathrm{s}} \cdot G \int_{N_{4}}^{+\infty} \frac{1}{\theta_{2}} \cdot p\left(\frac{z-R_{1} \cdot \theta_{1}-d \cdot \theta_{3}}{\theta_{2}}\right) d z \\
& \text { S.t. } N_{3} \geq G \\
& \begin{array}{l}
\int_{-\infty}^{M R} \frac{1}{\theta_{2}} \cdot p\left(\frac{z-R_{1} \cdot \theta_{1}-e \cdot \theta_{3}}{\theta_{2}}\right) d z \leq \sigma \\
\theta_{1}+\theta_{2}+\theta_{3}=1
\end{array} \\
& 0 \leq \theta_{i} \leq 1, \quad i \in\{1,2,3\}
\end{aligned}
$$

Formula (4) describes the objective of portfolio optimization, that is, maximizing the TRP value. Formula (5) is the risk constraint of investors, 
A Portfolio Optimization Perspective Based on Tri-Reference Point Theory for Explaining the Attractiveness of Structured Products

indicating that the probability of a return below the MR is controlled within a preset probability. Formula (6) makes the total investment proportion equal 1.

\section{Algorithm design}

Because the objective functions in section 3.2 contain limits of integrals associated with the unknown decision variables, the conventional algorithms cannot be directly used to solve the models in section 3.2. Inspired by Zhang et al. (2016), in algorithm 1, we utilize the gradient information of the objective functions and the nonlinear constraints to transform the original models into the linear programming models to obtain the approximate optimal solution. However, the efficiency of this algorithm depends on the setting of the initial solution. If the initial solution is set inappropriately, there are too many iterations, which takes a long running time. To improve this point, we combine this algorithm with the particle swarm optimization (PSO) algorithm (Kennedy \& Eberhart, 1995) to form a new algorithm. The new algorithm is more stable than the PSO and does not depend on the initial settings.

The characteristics of our proposed algorithm cannot satisfy the equality constraint (6), and thus we need to replace the investment proportion constraints (6) and (7) with formulas (8) and (9) in all cases. In addition, equation (10) is substituted into the objective functions and constraints in section 3.2 to eliminate the decision variable $\theta_{3}$ :

$$
\begin{gathered}
0 \leq \theta_{1} \leq 1 \\
0 \leq \theta_{2} \leq 1-\theta_{1} \\
\theta_{3}=1-\theta_{1}-\theta_{2}
\end{gathered}
$$

\subsection{Algorithm 1}

Algorithm 1 is designed based on the minimum gradient information calculated according to formula (11):

$$
\frac{\partial}{\partial \theta_{i}} \int_{M\left(\theta_{i}\right)}^{N\left(\theta_{i}\right)} f\left(z, \theta_{i}\right) d z=\int_{M\left(\theta_{i}\right)}^{N\left(\theta_{i}\right)} \frac{\partial f\left(z, \theta_{i}\right)}{\partial \theta_{i}} d z+f\left(N\left(\theta_{i}\right), \theta_{i}\right) \frac{d N\left(\theta_{i}\right)}{d t}-f\left(M\left(\theta_{i}\right), \theta_{i}\right) \frac{d M\left(\theta_{i}\right)}{d t}
$$

For a given $\boldsymbol{\theta}$, the gradient information of $F(\boldsymbol{\theta})$ in Section 3.2 and $I(\boldsymbol{\theta})$ in formula (5) are obtained by formula (11).

Because the portfolio payoff function of $\mathrm{P} 2$ is more complex than that of $\mathrm{P} 1$, the model of Case 5 of $\mathrm{P} 2$ is taken as an example. The steps of algorithm 1 are given as follows:

Step 1. Give an initial feasible solution $\boldsymbol{\theta}^{0}=\left(\theta_{1}^{0}, \theta_{2}^{0}\right)$ and $V^{0}>0$ large enough. $\delta>0$ is the required precision. Set iteration $\mathrm{k}=0$.

DOI: 10.24818/18423264/54.1.20.12 
Step 2. If $\left|V^{k}\right|<\delta$, the algorithm stops, and the results are output. Otherwise, go to step 3.

Step 3. Solve a subproblem with the given $\boldsymbol{\theta}^{k}$; then obtain $V^{k}$ and the search direction $\boldsymbol{D}^{k}=\left(D_{1}^{k}, D_{2}^{k}\right)$. The subproblem model is as below.

$$
\begin{gathered}
\operatorname{Min} V^{k} \\
\text { S.t. } \nabla F_{5}\left(\boldsymbol{\theta}^{k}\right)^{T} \boldsymbol{D}^{k}-V^{k} \leq 0 \\
-\left(\theta_{1}^{k}+D_{1}^{k}\right)-V^{k} \leq 0 \\
\left(\theta_{1}^{k}+D_{1}^{k}\right)-V^{k} \leq 1 \\
-\left(\theta_{2}^{k}+D_{2}^{k}\right)-V^{k} \leq 0 \\
\left(\theta_{1}^{k}+D_{1}^{k}\right)+\left(\theta_{2}^{k}+D_{2}^{k}\right)-V^{k} \leq 1 \\
e+\left(R_{1}-e\right)\left(\theta_{1}^{k}+D_{1}^{k}\right)-V^{k} \leq G \\
-\left[d+\left(R_{1}-d\right)\left(\theta_{1}^{k}+D_{1}^{k}\right)\right]-V^{k} \leq-G \\
I\left(\boldsymbol{\theta}^{k}\right)+\nabla I\left(\boldsymbol{\theta}^{k}\right)^{T} \boldsymbol{D}^{k}-V^{k} \leq \sigma
\end{gathered}
$$

where $\nabla F_{5}\left(\boldsymbol{\theta}^{k}\right)$ is the gradient vector of $F_{5}(\boldsymbol{\theta})$ in iteration k. $\nabla I\left(\boldsymbol{\theta}^{k}\right)$ is the gradient vector of $I(\theta)$ in iteration $\mathrm{k}$.

Step 4. With the obtained $\boldsymbol{D}^{k}$, calculate the maximum step length $\alpha_{k}^{\max }$ using the following linear programming model:

$$
\begin{gathered}
\text { Min }-\alpha_{k}^{\max } \\
\text { S.t. } \theta_{1}^{k}+\alpha_{k}^{\max } \cdot D_{1}^{k} \geq 0 \\
\theta_{1}^{k}+\alpha_{k}^{\max } \cdot D_{1}^{k} \leq 1 \\
\theta_{2}^{k}+\alpha_{k}^{\max } \cdot D_{2}^{k} \geq 0 \\
\theta_{1}^{k}+\alpha_{k}^{\max } \cdot D_{1}^{k}+\theta_{2}^{k}+\alpha_{k}^{\max } \cdot D_{2}^{k} \leq 1 \\
e+\left(R_{1}-e\right)\left(\theta_{1}^{k}+\alpha_{k}^{\max } \cdot D_{1}^{k}\right) \leq G \\
d+\left(R_{1}-d\right)\left(\theta_{1}^{k}+\alpha_{k}^{\max } \cdot D_{1}^{k}\right) \geq G \\
I\left(\boldsymbol{\theta}^{\mathrm{k}}\right)+\alpha_{k}^{\max } \cdot \nabla I\left(\boldsymbol{\theta}^{k}\right)^{T} \boldsymbol{D}^{k} \leq \sigma
\end{gathered}
$$

Step 5. Compute the feasible step length $\alpha_{k}$ by solving

$$
-F_{5}\left(\boldsymbol{\theta}^{\mathrm{k}}+\alpha_{k} \cdot \boldsymbol{D}^{k}\right) \leq-F_{5}\left(\boldsymbol{\theta}^{k}\right)-\lambda \alpha_{k} \cdot \nabla F\left(\boldsymbol{\theta}_{k}\right)^{T} \boldsymbol{D}_{k}
$$

where $\alpha_{k}=\eta^{j} \alpha_{k}^{\max }, j \in\{0,1,2, \cdots\}$ is the minimal integer that makes the above inequality true, and $0<\eta<1$ and $0<\lambda<1$ are given constants.

Step 6. Set $\boldsymbol{\theta}^{\mathrm{k}+1}=\boldsymbol{\theta}^{k}+\alpha_{k} \cdot \boldsymbol{D}_{k}$ and $k=k+1$. Return to step 2. 
A Portfolio Optimization Perspective Based on Tri-Reference Point Theory for Explaining the Attractiveness of Structured Products

\subsection{Particle swarm optimization algorithm}

PSO is a kind of evolutionary computation method based on swarm intelligence. It is characterized by its simplicity and easy implementation. In PSO, particles composed of one-dimensional vectors are used to represent the asset proportions, and the feasible solutions are obtained by setting their respective representations for the velocities and positions of the particles and giving them update criteria. Combining PSO with the characteristics of the portfolio optimization problem, the following steps are taken:

Step 1. Give the number of particles, randomize the positions and velocities of each particle, and set the total number of iterations.

Step 2. Calculate the objective function values of the portfolio optimization problem based on the positions of particles, also called fitness values.

Step 3. Under the premise of meeting constraint (5), the fitness values are used to measure the quality of solutions.

Step 4. Change the velocities and positions of particles according to formulas (12) and (13).

Step 5. If the preset number of iterations is reached, the position of the optimal particle and the objective function value are output; otherwise, return to step 2.

Particle representation: Each particle's position, denoted as $\boldsymbol{X}=\left(\theta_{1}, \theta_{2}\right)$, is a feasible solution, and this particle's velocity is $\boldsymbol{Y}=\left(y_{1}, y_{2}\right)$.

Initialization: Under the constraints of formulas (8) and (9), the positions of particles are randomly initialized, and the velocities of the particles are randomly initialized in the interval $(-0.05,0.05)$. If the initial solution does not satisfy constraint (5), it is reinitialized.

Iterative optimization: In each iteration, the particles are updated by tracking two "optimums": The first is that found by a given particle. The second is that found by all the particles. Because our goal is to maximize the perceived value under constraint (5), the update is performed when the objective function value becomes larger and the constraint is satisfied. In the iterative process, if the positions and velocities of the particles exceed the value ranges mentioned in the above initialization process, the boundary values will be taken. The particle position and velocity update formulas are as follows:

$$
\begin{gathered}
\boldsymbol{Y}_{h}^{k+1}=\omega \times \boldsymbol{Y}_{h}^{k}+C_{1} \times \operatorname{random}(0,1) \times\left(\boldsymbol{X}_{h p}^{k}-\boldsymbol{X}_{h}^{k}\right)+C_{2} \times \operatorname{random}(0,1) \times\left(\boldsymbol{X}_{g}^{k}-\boldsymbol{X}_{h}^{k}\right) \\
\boldsymbol{X}_{h}^{k+1}=\boldsymbol{X}_{h}^{k}+\boldsymbol{Y}_{h}^{k+1}(13)
\end{gathered}
$$

where $k$ is the iteration counter, $\boldsymbol{Y}_{h}^{k}$ is the velocity of particle $h$ in iteration $k, \boldsymbol{X}_{h}^{k}$ is the position of particle $h$ in iteration $k, X_{h p}^{k}$ is the best position reached by particle $h$ until iteration $k, X_{g}^{k}$ is the best position reached by all the particles until iteration

DOI: 10.24818/18423264/54.1.20.12 
$k, C_{1}$ and $C_{2}$ are cognitive and social parameters that are set to 1 , and $\omega$ is the inertia weight. Generally, the initial value of $\omega$ is set to 0.9 , and then $\omega$ decreases linearly as iteration times increase until $\omega=0.4$

\subsection{Combination of algorithm 1 and PSO}

In order to guarantee the speed and accuracy of the computation at the same time by combining algorithm 1 with PSO, the new algorithm is implemented as follows:

Step 1. Give the number of particles, randomize the positions and velocities of each particle, and set $V^{0}>0$ and $\delta>0$.

Step 2. Calculate the objective function $F_{5}(\theta)$ values of the portfolio optimization problem based on the positions of the particles.

Step 3. Compare the fitness values of the particles under the premise of meeting constraint (5). Then, the position of the particle with the maximum fitness value is taken as the initial solution of algorithm 1 , and $V^{k}$ and $\boldsymbol{\theta}^{k+1}$ are obtained.

Step 4. If $\left|V^{k}\right|<\delta$, the algorithm stops, and the results are output. Otherwise, go to step 5.

Step 5. Change the velocities and positions of the other particles according to formulas (12) and (13). Then, combine the updated particles in this step with the updated particle in step 3. Return to step 2.

\section{Optimization results and analysis}

With the objective of maximizing the TRP value under the constraint of the safety-first principle, the influence of different parameters on two portfolios' components is analyzed by changing the values of MR and G in TRP theory. The reasons and preconditions for structured products attracting investors are discussed.

\subsection{Portfolio P1 including structured product $3 \mathrm{~A}$}

The weights of the four regions of failure, loss, gain and success are 4, 2, 1, and 3 , respectively, and the tolerance ois 0.05 . For $\mathrm{P} 1$, the changes in the investment proportions with different MR and G values are shown in Tables 1 and 2. To fully reflect the change trend, the initial $G$ is increased stepwise by 0.005 . To avoid redundancy, $G$ value intervals are gradually increased in the later stages. According to Tables 1 and 2, when the value of MR is the same and that of G is no more than $7 \%$, the proportion of asset $3 \mathrm{~A}$ increases gradually, that of asset 1 decreases gradually and that of underlying asset 2 is always 0 . In fact, for investors, avoiding failure (being below MR) is the most important, followed by success (being above $\mathrm{G}$ ). Thus, when the $\mathrm{G}$ value is not particularly large, they will try to avoid asset 2, which is then most likely to fail. Between assets 1 and $3 \mathrm{~A}$, there is no possibility of failure. Success is most important at this point, and only $3 \mathrm{~A}$ provides the opportunity to achieve G, so as the gap between $\mathrm{G}$ and the fixed return of asset 
A Portfolio Optimization Perspective Based on Tri-Reference Point Theory for Explaining the Attractiveness of Structured Products

1 widens, investors will increasingly favor asset $3 \mathrm{~A}$. When $\mathrm{G}$ exceeds $7 \%$, the upper limit of 3A, as $3 \mathrm{~A}$ cannot achieve success, investors began to gradually shift to asset 2, reducing investment in 3A. Magron (2014) shows that high-aspiration investors hold riskier portfolios, and our results come to the same conclusion by a different route. As shown in Tables 1 and 2, when $\mathrm{G}$ is fixed, in the portfolios including asset 2, as MR falls, the risk that investors can tolerate increases, and the proportion of asset 2 that might fail increases. Therefore, asset $3 \mathrm{~A}$ is best suited to investors with relatively but not very high MR and G. Moreover, when G fixed, in the portfolios including asset 2, the maximum TRP value increases as MR declines. This is because the probability of falling into the failure region decreases.

Table 1. Investment proportions in $P 1$ with different $G$ values, $M R=-0.05,-0.1$

\begin{tabular}{|c|c|c|c|c|c|c|c|c|}
\hline \multirow{3}{*}{ G } & \multicolumn{8}{|c|}{ MR } \\
\hline & \multicolumn{4}{|c|}{-0.05} & \multicolumn{4}{|c|}{-0.1} \\
\hline & Asset 1 & Asset 2 & Asset 3A & TRP value & Asset 1 & Asset 2 & Asset $3 \mathrm{~A}$ & TRP value \\
\hline 0.055 & 0.75 & 0 & 0.25 & 0.105 & 0.75 & 0 & 0.25 & 0.105 \\
\hline 0.06 & 0.5 & 0 & 0.5 & 0.11 & 0.5 & 0 & 0.5 & 0.11 \\
\hline 0.065 & 0.25 & 0 & 0.75 & 0.115 & 0.25 & 0 & 0.75 & 0.115 \\
\hline 0.07 & 0 & 0 & 1 & 0.12 & 0 & 0 & 1 & 0.12 \\
\hline 0.075 & 0 & 0.1566 & 0.8434 & 0.1187 & 0 & 0.1599 & 0.8401 & 0.1189 \\
\hline 0.08 & 0 & 0.2186 & 0.7814 & 0.1162 & 0 & 0.2180 & 0.7820 & 0.1165 \\
\hline 0.09 & 0 & 0.3235 & 0.6765 & 0.1153 & 0 & 0.3084 & 0.6916 & 0.1148 \\
\hline 0.1 & 0 & 0.3235 & 0.6765 & 0.1139 & 0 & 0.3935 & 0.6065 & 0.1146 \\
\hline 0.12 & 0 & 0.3235 & 0.6765 & 0.1053 & 0 & 0.5256 & 0.4744 & 0.1170 \\
\hline 0.15 & 0.6259 & 0.3741 & 0 & 0.0909 & 0.4389 & 0.5611 & 0 & 0.1131 \\
\hline 0.2 & 0.6259 & 0.3741 & 0 & 0.0747 & 0.4389 & 0.5611 & 0 & 0.0985 \\
\hline
\end{tabular}

Table 2. Investment proportions in $P 1$ with different $G$ values, $M R=-0.15,-0.2$

\begin{tabular}{|c|c|c|c|c|c|c|c|c|}
\hline \multirow{3}{*}{ G } & \multicolumn{8}{|c|}{ MR } \\
\hline & \multicolumn{4}{|c|}{-0.15} & \multicolumn{4}{|c|}{-0.2} \\
\hline & Asset 1 & Asset 2 & Asset 3A & TRP value & Asset 1 & Asset 2 & Asset 3A & TRP value \\
\hline 0.055 & 0.75 & 0 & 0.25 & 0.105 & 0.75 & 0 & 0.25 & 0.105 \\
\hline 0.06 & 0.5 & 0 & 0.5 & 0.11 & 0.5 & 0 & 0.5 & 0.11 \\
\hline 0.065 & 0.25 & 0 & 0.75 & 0.115 & 0.25 & 0 & 0.75 & 0.115 \\
\hline 0.07 & 0 & 0 & 1 & 0.12 & 0 & 0 & 1 & 0.12 \\
\hline 0.075 & 0 & 0.1618 & 0.8382 & 0.1190 & 0 & 0.1625 & 0.8375 & 0.1190 \\
\hline 0.08 & 0 & 0.2227 & 0.7773 & 0.1168 & 0 & 0.2252 & 0.7748 & 0.1169 \\
\hline 0.09 & 0 & 0.3137 & 0.6863 & 0.1154 & 0 & 0.3200 & 0.6800 & 0.1158 \\
\hline 0.1 & 0 & 0.3905 & 0.6095 & 0.1154 & 0 & 0.3983 & 0.6017 & 0.1161 \\
\hline 0.12 & 0 & 0.5394 & 0.4606 & 0.1169 & 0 & 0.5375 & 0.4625 & 0.1179 \\
\hline 0.15 & 0 & 0.7278 & 0.2722 & 0.1218 & 0 & 0.7531 & 0.2469 & 0.1218 \\
\hline 0.2 & 0.2518 & 0.7482 & 0 & 0.1182 & 0.0649 & 0.9351 & 0 & 0.1289 \\
\hline
\end{tabular}

The essence of TRP theory is reflected in the different degree of importance among the weights of the four regions. This emphasizes the importance of the failure and success regions. To determine the influence of specific weight values on investment proportions, the weights of failure and success are changed to observe the change in investment proportion without changing the size relationship

DOI: 10.24818/18423264/54.1.20.12 
among the weights. Tables 3 and 4 show the change in the investment proportion with respect to $\mathrm{G}$ when $\beta_{S}$ and $\beta_{F}$ vary. MR is set at -0.05 , and $\sigma$ is $0.05 . \beta_{F}=4$ in Table 3 , and $\beta_{S}=3$ in Table 4 . Tables 3 and 4 indicate that according to TRP theory, under the premise that the failure weight is greater than the success weight, the success weight is greater than the loss weight, and the loss weight is greater than the gain weight, the proportion of the underlying always increases with an increase in $G$ when $G$ reaches a certain level, regardless of how the specific value of the region weight changes. Moreover, investors will be more inclined to take risks, which also demonstrates the stability of our conclusion. From Table 3, the proportion of asset 2 increases as the success weight increases, indicating that the more eager investors are, the more venturous they are. In contrast to Table 3, Table 4 shows that the proportion of asset 2 decreases as the failure weight increases, indicating that people who are more concerned about failure tend to be more conservative in their investment activities.

Table 3. Investment proportions in P1 with different $G$ values as the success weight varies

\begin{tabular}{|c|c|c|c|c|c|c|c|c|c|}
\hline \multirow{3}{*}{ G } & \multicolumn{9}{|c|}{$\beta_{S}$} \\
\hline & \multicolumn{3}{|c|}{3} & \multicolumn{3}{|c|}{3.4} & \multicolumn{3}{|c|}{3.8} \\
\hline & Asset 1 & Asset 2 & Asset 3A & Asset 1 & Asset 2 & Asset $3 \mathrm{~A}$ & Asset 1 & Asset 2 & Asset 3A \\
\hline 0.075 & 0 & 0.1566 & 0.8434 & 0 & 0.1667 & 0.8333 & 0 & 0.1667 & 0.8333 \\
\hline 0.08 & 0 & 0.2186 & 0.7814 & 0 & 0.2388 & 0.7612 & 0 & 0.2584 & 0.7416 \\
\hline 0.085 & 0 & 0.2793 & 0.7207 & 0 & 0.3113 & 0.6887 & 0 & 0.3235 & 0.6765 \\
\hline
\end{tabular}

Table 4. Investment proportions in $P 1$ with different $G$ values as the failure weight varies

\begin{tabular}{|c|c|c|c|c|c|c|c|c|c|}
\hline \multirow{3}{*}{ G } & \multicolumn{9}{|c|}{$\beta_{F}$} \\
\hline & \multicolumn{3}{|c|}{4} & \multicolumn{3}{|c|}{4.4} & \multicolumn{3}{|c|}{4.8} \\
\hline & Asset 1 & Asset 2 & Asset 3A & Asset 1 & Asset 2 & Asset $3 \mathrm{~A}$ & Asset 1 & Asset 2 & Asset $3 \mathrm{~A}$ \\
\hline 0.075 & 0 & 0.1566 & 0.8434 & 0 & 0.1543 & 0.8457 & 0 & 0.1522 & 0.8478 \\
\hline 0.08 & 0 & 0.2186 & 0.7814 & 0 & 0.2126 & 0.7874 & 0 & 0.2074 & 0.7926 \\
\hline 0.085 & 0 & 0.2793 & 0.7207 & 0 & 0.2676 & 0.7324 & 0 & 0.2581 & 0.7419 \\
\hline
\end{tabular}

\subsection{Portfolio $P 2$ including structured product $3 B$}

The weights of the four regions of failure, loss, gain and success are 4, 2, 1, and 3 , respectively. The tolerance $\sigma$ is 0.05 . For P2, the changes in the investment proportions with different MR and G are shown in Tables 5 and 6 . The two tables indicate that the trend of the change in the investment proportion is similar to that of P1. Specifically, when G is lower than the upper limit of structured product 3B, the proportion of $3 \mathrm{~B}$ increases gradually; whereas when $\mathrm{G}$ is higher than the upper limit, the proportion of $3 \mathrm{~B}$ decreases, and the proportion of the underlying increases to meet the goal needs of investors. Although the trend of portfolios P2 and P1 is the same, there are some differences in the specific values. Combined 
A Portfolio Optimization Perspective Based on Tri-Reference Point Theory for Explaining the Attractiveness of Structured Products

with Tables $1,2,5$, and 6 , the proportion of $3 \mathrm{~B}$ is greater than that of $3 \mathrm{~A}$ when $\mathrm{G}$ and MR are the same, and the maximum TRP value of portfolio $\mathrm{P} 2$ is greater than that of $\mathrm{P} 1$ before $\mathrm{G}$ reaches 0.0621 , the upper limit payoff of 3B. This shows that investors with smaller $\mathrm{G}$ prefer $3 \mathrm{~B}$ to $3 \mathrm{~A}$. When $\mathrm{G}$ is greater than 0.07 , the upper limit payoff of 3A,the proportion of $3 \mathrm{~B}$ is less than that of $3 \mathrm{~A}$, and the maximum TRP value of $\mathrm{P} 2$ is less than that of $\mathrm{P} 1$, indicating that investors with higher $\mathrm{G}$ prefer $3 \mathrm{~A}$.

Table 5. Investment proportions in $P 2$ with different $G$ values, $M R=-0.05,-0.1$

\begin{tabular}{|c|c|c|c|c|c|c|c|c|}
\hline \multirow{3}{*}{ G } & \multicolumn{8}{|c|}{ MR } \\
\hline & \multicolumn{4}{|c|}{-0.05} & \multicolumn{4}{|c|}{-0.1} \\
\hline & Asset 1 & Asset 2 & Asset 3B & TRP value & Asset 1 & Asset 2 & Asset 3B & TRP value \\
\hline 0.055 & 0.5806 & 0 & 0.4194 & 0.1147 & 0.5806 & 0 & 0.4194 & 0.1147 \\
\hline 0.06 & 0.1736 & 0 & 0.8264 & 0.1206 & 0.1736 & 0 & 0.8264 & 0.1206 \\
\hline 0.0621 & 0 & 0 & 1 & 0.1230 & 0 & 0 & 1 & 0.1230 \\
\hline 0.07 & 0 & 0.1807 & 0.8193 & 0.1114 & 0 & 0.1833 & 0.8167 & 0.1117 \\
\hline 0.075 & 0 & 0.2347 & 0.7653 & 0.1103 & 0 & 0.2316 & 0.7684 & 0.1107 \\
\hline 0.08 & 0 & 0.2910 & 0.7090 & 0.1102 & 0 & 0.2740 & 0.7260 & 0.1102 \\
\hline 0.09 & 0 & 0.3235 & 0.6765 & 0.1107 & 0 & 0.3538 & 0.6462 & 0.1104 \\
\hline 0.1 & 0 & 0.3235 & 0.6765 & 0.1088 & 0 & 0.4379 & 0.5621 & 0.1115 \\
\hline 0.12 & 0.6259 & 0.3741 & 0 & 0.1033 & 0.4389 & 0.5611 & 0 & 0.1150 \\
\hline 0.15 & 0.6259 & 0.3741 & 0 & 0.0909 & 0.4389 & 0.5611 & 0 & 0.1131 \\
\hline 0.2 & 0.6259 & 0.3741 & 0 & 0.0747 & 0.4389 & 0.5611 & 0 & 0.0985 \\
\hline
\end{tabular}

Table 6. Investment proportions in $P 2$ with different $G$ values, $M R=-0.15,-0.2$

\begin{tabular}{|c|c|c|c|c|c|c|c|c|}
\hline \multirow{3}{*}{ G } & \multicolumn{8}{|c|}{ MR } \\
\hline & \multicolumn{4}{|c|}{-0.15} & \multicolumn{4}{|c|}{-0.2} \\
\hline & Asset 1 & Asset 2 & Asset 3B & TRP value & Asset 1 & Asset 2 & Asset 3B & TRP value \\
\hline 0.055 & 0.5806 & 0 & 0.4194 & 0.1147 & 0.5806 & 0 & 0.4194 & 0.1147 \\
\hline 0.06 & 0.1736 & 0 & 0.8264 & 0.1206 & 0.1736 & 0 & 0.8264 & 0.1206 \\
\hline 0.0621 & 0 & 0 & 1 & 0.1230 & 0 & 0 & 1 & 0.1230 \\
\hline 0.07 & 0 & 0.1861 & 0.8139 & 0.1119 & 0 & 0.1873 & 0.8127 & 0.1119 \\
\hline 0.075 & 0 & 0.2365 & 0.7635 & 0.1110 & 0 & 0.2394 & 0.7606 & 0.1111 \\
\hline 0.08 & 0 & 0.2797 & 0.7203 & 0.1107 & 0 & 0.2844 & 0.7156 & 0.1110 \\
\hline 0.09 & 0 & 0.3559 & 0.6441 & 0.1112 & 0 & 0.3631 & 0.6369 & 0.1117 \\
\hline 0.1 & 0 & 0.4268 & 0.5732 & 0.1121 & 0 & 0.4336 & 0.5664 & 0.1129 \\
\hline 0.12 & 0 & 0.5731 & 0.4269 & 0.1149 & 0 & 0.5665 & 0.4335 & 0.1158 \\
\hline 0.15 & 0.2518 & 0.7482 & 0 & 0.1212 & 0.2087 & 0.7913 & 0 & 0.1212 \\
\hline 0.2 & 0.2518 & 0.7482 & 0 & 0.1182 & 0.0648 & 0.9352 & 0 & 0.1289 \\
\hline
\end{tabular}

\section{Conclusions}

Portfolio selection essentially belongs to the topic of risk choice. TRP theory provides qualitative and quantitative perspectives for the field of risk choice. We introduce TRP theory into the portfolio optimization problem. Motivated by the flourishing of structured products, we analyze investors' decision-making behavior from three psychological points and four perceptual regions composed of MR, SQ and G. In addition, we develop a hybrid particle swarm optimization

DOI: 10.24818/18423264/54.1.20.12 
algorithm to solve the portfolio selection model with an objective function that includes limits of integrals with unknown decision variables.

We find that the payoff characteristics of interval structured products play a decisive role in the attractiveness of those products. On the one hand, the lower limit reduces the possibility of failure and makes structured products preferable to the underlying assets from a risk perspective. On the other hand, the upside potential provides investors with a chance for success and enables them to outperform low-risk assets in terms of return. Consequently, it is no accident that China's existing structured products attract investors; rather, their popularity is inevitable because investors with a relatively high MR and G are widespread in the market.

This paper establishes the portfolio selection models that are applicable only when investors have accurate and fixed knowledge of their own MR, SQ and G. However, it is reasonable to imagine that investors' reference points may fluctuate flexibly with the outside world, such as their realized payoff in previous investing activities. Moreover, a small number of investors may have a vague understanding of their reference points. All of the abovementioned factors can be considered in future work. Besides, the proposed algorithm can also be applied to other specific portfolio optimization problems.

\section{ACKNOWLEDGMENTS}

The first author is grateful to the National Natural Science Foundation of China (No. 71631008) for financial support.

\section{REFERENCES}

[1]Abreu, M. \& Mendes, V. (2018), The Investor in Structured Retail Products: Advice Driven or Gambling Oriented. Journal of Behavioral and Experimental Finance, 17, 1-9;

[2]Allais, M. (1953), Le comportement de l'hommerationneldevant le risque: critique des postulats et axiomes de l'ecoleamericaine. Econometrica, 21(4), 503-546;

[3]Cao, J. (2017), How Does the Underlying Affect the Risk-return Profiles of Structured Products? Financial Markets \& Portfolio Management, 31(1), 27-47; [4]Chiu, M. C., Wong, H. Y. \& Zhao, J. (2018), Dynamic Safety First Expected Utility Model. European Journal of Operational Research, 271(1), 141-154; [5]Döbeli, B. \&Vanini, P. (2010), Stated and Revealed Investment Decisions Concerning Retail Structured Products. Journal of Banking \& Finance, 34(6), 1400-1411;

[6]Entrop, O., Mckenzie, M., Wilkens, M. \& Winkler, C. (2016), The Performance of Individual Investors in Structured Financial Products. Review 
A Portfolio Optimization Perspective Based on Tri-Reference Point Theory for Explaining the Attractiveness of Structured Products

of Quantitative Finance \& Accounting, 46(3), 569-604;

[7]Gao, J., Xiong, Y. \& Li, D. (2016), Dynamic Mean-Risk Portfolio Selection with Multiple Risk Measures in Continuous-Time. European Journal of Operational Research, 249(2), 647-656;

[8]Harris, C. \& Wu, C. (2014), Using Tri-Reference Point Theory to Evaluate Risk Attitude and the Effects of Financial Incentives in a Gamified Crowdsourcing Task. Journal of Business Economics, 84(3), 281-302; [9]Hens, T. \& Rieger, M. O. (2008), The Dark Side of the Moon: Structured Products from the Customer's Perspective. Working Paper;

[10]Hens, T. \& Rieger, M. O. (2014), Can Utility Optimization Explain the Demand for Structured Investment Products. Quantitative Finance, 14(4), 673-681;

[11]Hu, Z. \& Wang, X. T. (2014), Trust or not: Heuristics for Making Trustbased Choices in hr Management. Journal of Business Research, 67(8), 17101716 ;

[12]Kahneman, D. \& Tversky, A. (1979), Prospect Theory: An analysis of Decisions under Risk. Econometrica, 47, 263-292;

[13]Keemun, W. \& Musa, G. (2015), International Second Home Retirement Motives in Malaysia: Comparing British and Japanese Retirees. Asia Pacific Journal of Tourism Research, 20(9), 1041-1062;

[14]Kennedy, J. \& Eberhart, R. (1995), Particle Swarm Optimization. IEEE international conference on neural networks IV, 4(8), 1942-1948;

[15]Kiriakopoulos, K. \&Mavralexakis, T. (2011), Structured Bonds and Greek Demons-Is the Attack "Fair". Journal of Applied Finance \& Banking, 1(2), 231277;

[16]Lagerkvist, C. J., Normann, A. \& Åström, A. (2015), A Theoretical Description and Experimental Exploration of Tri-Reference Point Theory with Respect to Food Choice. Food Quality \& Preference, 41, 60-74;

[17]Levy, H. \& Levy, M. (2009), The Safety First Expected Utility Model: Experimental Evidence and Economic Implications. Journal of Banking \& Finance, 33(8), 1494-1506;

[18]Liagkouras, K. (2019), A New Three-Dimensional Encoding Multiobjective Evolutionary Algorithm with Application to the Portfolio Optimization Problem. Knowledge-Based Systems, 163, 186-203;

[19]Lopes, L. L. (1987), Between Hope and Fear: The Psychology of Risk. Advances in Experimental Social Psychology, 20(3), 255-295;

[20]Macedo, L. L., Godinho, P. \& Alves, M. J. (2017), Mean-Semivariance Portfolio Optimization with Multiobjective Evolutionary Algorithms and Technical Analysis Rules. Expert Systems with Applications, 79, 33-43; [21]Magron, C. (2014), Investors' Aspirations and Portfolio Performance. Finance Research Letters, 11(2), 153-160; [22]Markowitz, H. (1952), Portfolio Selection. Journal of Finance, 7(1), 77-91; [23]Nalpas, N., Simar, L. \&Vanhems, A. (2017), Portfolio Selection in a Multi-

DOI: 10.24818/18423264/54.1.20.12 
Zongrun Wang, Tangtang He, Xuekai Ni

Moment Setting: A Simple Monte-Carlo-FDH Algorithm. European Journal of Operational Research, 263(1), 308-320;

[24]Pyle, D. \&Turnovsky, S. (1970), Safety-First and Expected Utility

Maximization in Mean-Standard Deviation Portfolio Analysis. The Review of Economics and Statistics, 52(1), 75-81;

[25]Rieger, M. O. (2012), Why Do Investors Buy Bad Financial Products?

Probability Misestimation and Preferences in Financial Investment

Decision. Journal of Behavioral Finance, 13(2), 108-118;

[26]Rieger, M. O. \& Hens, T. (2012), Explaining the Demand for Structured

Financial Products: Survey and Field Experiment Evidence. Zeitschrift Für

Betriebswirtschaft, 82(5), 491-508;

[27]Roy, A. D. (1952), Safety First and the Holding of Assets. Econometrica, 20(3), 431-449;

[28]Tversky, A. \& Kahneman, D. (1992), Advances in Prospect Theory:

Cumulative Representation of Uncertainty. Journal of Risk \& Uncertainty, 5(4), 297-323;

[29]Vandenbroucke, J. (2015), A Cumulative Prospect View on Portfolios that

Hold Structured Products. Journal of Behavioral Finance, 16(4), 297-310;

[30]Wang, X. T. \& Johnson, J. G. (2012), A Tri-Reference Point Theory of

Decision Making under Risk. Journal of Experimental Psychology:

General, 141(4), 743-756;

[31]Yao, H., Li, Y. \& Benson, K. (2015), A Smooth Non-Parametric Estimation

Framework for Safety-First Portfolio Optimization. Quantitative Finance, 15(11), $1865-1884$

[32]Zhang, X., Huang, S. \& Wan, Z. (2016), Optimal Pricing and Ordering in Global Supply Chain Management with Constraints under Random Demand.

Applied Mathematical Modelling, 40(23), 10105-10130;

[33]Zhao, L., Ye, J., Wu, X. \& Hu, F. (2018), The Influence of the Tri-

Reference Points on Fairness and Satisfaction Perception. Frontiers in

Psychology, 9, 193. 